\title{
BMJ Open Prognostic factors for acute exacerbation of idiopathic pulmonary fibrosis: protocol for a systematic review and meta-analysis
}

Hiroyuki Kamiya, ${ }^{1}$ Ogee Mer Panlaqui ${ }^{2}$

To cite: Kamiya H, Panlaqui OM. Prognostic factors for acute exacerbation of idiopathic pulmonary fibrosis: protocol for a systematic review and meta-analysis. BMJ Open 2019:9:e028226. doi:10.1136/ bmjopen-2018-028226

- Pre-publication history and additional material is published online only. To view please visit the journal online (http://dx.doi. org/10.1136/bmjopen-2018028226).

Received 27 November 2018 Revised 21 March 2019 Accepted 23 April 2019
Check for updates

(C) Author(s) (or their employer(s)) 2019. Re-use permitted under CC BY-NC. No commercial re-use. See rights and permissions. Published by BMJ.

${ }^{1}$ Occupational Respiratory Epidemiology, School of Population and Global Health, University of Western Australia, Perth, Western Australia,

Australia

${ }^{2}$ Department of Intensive Care Medicine, Northern Hospital, Melbourne, Victoria, Australia

Correspondence to Dr Hiroyuki Kamiya; mlb04194@nifty.com

\section{ABSTRACT}

Introduction Idiopathic pulmonary fibrosis (IPF) is a form of chronic fibrosing interstitial pneumonia with unknown disease aetiology. Acute exacerbation (AE) of IPF is an accelerated disease progression beyond its expected course. A 30 -day mortality of AE of IPF is $40 \%$. While death may occur, there is much variation in the clinical progression of this condition. Previous attempts have been made to investigate various possible prognostic factors for AE of IPF; however, they have yet to be confirmed. The aim of this systematic review is to clarify these prognostic factors.

Methods and analysis In this review, AE of IPF is the condition of interest, which has been defined according to previously established diagnostic criteria. The primary outcomes of interest include short-term all-cause mortality and pulmonary-cause mortality. The secondary outcomes of interest include long-term mortality and hospital separation for the disease. Primary studies investigating prognostic factors for AE of IPF are eligible for inclusion in this review. All study types are permitted except case reports. Two reviewers will search electronic databases, such as Medline and EMBASE, from 2002 to the 1 April 2019 and extract data independently. Risk of bias in individual studies will be assessed using the Quality in Prognostic Studies tool. Meta-analysis will be conducted for univariate data if at least three studies report the effect of a specific prognostic factor using similar statistical methods. Multivariate results will be reported qualitatively. Subgroup analysis and sensitivity analysis will be considered with the aim of generalising findings to the clinical settings and drawing more robust conclusions. The Grades of Recommendation, Assessment, Development and Evaluation (GRADE) method will be applied to evaluate the quality of evidence for each prognostic factor. Ethics and dissemination Ethical approval will not be required. Results will be reported in a peer-reviewed scientific journal.

PROSPERO registration number CRD42018106172

\section{INTRODUCTION}

\section{Rationale}

Idiopathic pulmonary fibrosis (IPF) is a form of chronic fibrosing interstitial pneumonia with unknown disease aetiology. IPF is the most common type of pneumonia among

\section{Strengths and limitations of this study}

- This systematic review and meta-analysis will be the first addressing prognostic factors for acute exacerbation of idiopathic pulmonary fibrosis and be the foremost evidence for this potentially fatal disease as large-scale cohort studies investigating this disease may prove difficult.

- This study will focus on relevant clinical information, commonly used in clinical practice, which may facilitate the application of the review's findings to the clinical setting.

- There may be difficulty in combining the result due to substantial heterogeneity between studies.

idiopathic interstitial pneumonias (IIPs). ${ }^{1}$ IPF is a progressive disease that can result in death. A recent study in the USA found that patients over the age of 65 years had a median survival time of 3.8 years. ${ }^{2}$ Another study reported numerous complications resulting from IPF such as lung cancer, pneumonia, pulmonary embolism and heart failure, with mortality often a consequence of respiratory failure due to IPF. ${ }^{3}$ However, after a rapid deterioration of IPF beyond its usually expected clinical course was recognised as not uncommon phenomenon, ${ }^{4}$ this condition was termed as acute exacerbation (AE) of IPF and demonstrated to be a major cause of death of the disease. ${ }^{5}$ In early reports, $\mathrm{AE}$ of IPF was diagnosed by excluding known causes of disease deterioration, in particular, eliminating potentially causative infectious agents. ${ }^{6}$ However, the latest international guideline proposed a new diagnostic criteria for AE of IPF, which isolates worsening symptoms and newly emerging bilateral radiological opacities, rather than focusing on the aetiology of the exacerbation. ${ }^{7}$ Irrespective of the aetiology of this phenomenon, it can be fatal, with a 30-day mortality rate of $40 \%^{8}$ and a 1-year mortality of over $80 \% .^{9}$ The 
absence of effective treatment may explain the high rate of mortality. ${ }^{10}$

The clinical course of AE of IPF can vary and does not always lead to immediate death in affected individuals. ${ }^{11}$ Recently, some studies trialling antifibrotic agents suggested a promising preventive effect for disease progression from IPF. ${ }^{12}$ To better prevent against the harmful effects of AE of IPF, prognostic factors for the disease must be determined. Identifying these factors may help in tailoring specific treatment options to affected patients and better anticipate the consequence of this disease. Several studies have investigated diverse clinical information that could be related to the prognosis of $\mathrm{AE}$ of IPF. However, these studies have been limited by small sample sizes drawn from a single institution. ${ }^{13}{ }^{14}$ Furthermore, it seems unfeasible to conduct a large-scale cohort study to compensate for this shortcoming of previous research because unpredictable and lethal clinical course might prevent a recruitment of a sufficient number of participants. ${ }^{15}$ Due to the disparity of existing evidence, the aim of the proposed systematic review and meta-analysis is to clarify prognostic factors for AE of IPF. The results from this study will be the leading evidence available for this condition. The aim of this article is to rationalise the need for a systematic review of prognostic factors for $\mathrm{AE}$ of IPF and outline a proposed methodology for research integrity and transparency. Expected results of this study will not be discussed in this article.

\section{Research aims}

The aim of the proposed systematic review is to clarify prognostic factors for AE of IPF.

\section{METHODS AND ANALYSIS}

\section{Patient and public involvement}

There is no patient and public involvement in the whole process of conducting this research.

\section{Registration}

This protocol has already been registered with PROSPERO (International Prospective Register of Systematic Reviews). ${ }^{16}$

\section{Eligibility criteria}

\section{Subjects}

Patients with AE of IPF are eligible for this review. IPF will be diagnosed based on previously published international guidelines, such as an official American Thoracic Society/European Respiratory Society/Japanese Respiratory Society/Latin American Thoracic Association (ATS/ERS/JRS/ALAT) statement. ${ }^{17}$ AE of IPF will be diagnosed based on the latest international guideline, which consists of a previous or concurrent diagnosis of IPF, acute worsening or development of dyspnoea (typically within less than 1 month), newly emerging bilateral ground glass opacity (GGO) and/or consolidation superimposed on a background radiological change consistent with usual interstitial pneumonia (UIP) on high-resolution computed tomography (HRCT) scans. ${ }^{7}$ Although it is necessary to rule out cardiac failure or fluid overload as a cause of deterioration of IPF, infections or other potential triggers of AE of IPF do not need to be excluded, as per the latest diagnostic criteria, which accounts for both triggered and idiopathic cases. Accordingly, previously proposed diagnostic criteria (which emphasised the exclusion of pulmonary infection using endotracheal aspirate or bronchoalveolar lavage $)^{6}$ can be used to justify the inclusion of idiopathic cases for the disease under the latest diagnostic criteria. ${ }^{7}$ Subjects diagnosed with a rapid progressive form of interstitial pneumonia at their first presentation will also be included. However, their diagnosis must have been accompanied by radiological and/ or pathological UIP and known causes for the disease, such as connective tissue disease ${ }^{18}$ or drug toxicity must have been absent. ${ }^{19}$ In cases where patients had multiple episodes of AE of IPF, only the first presentation of the disease will be considered for further analysis.

\section{Exposures}

Any clinical information related to demographics, symptoms, pulmonary functions, radiological findings and laboratory tests will be considered as potential prognostic factors for AE of IPF, provided they have been investigated for their association with the outcomes of the disease. These factors may include age, sex, breathlessness, percentage of predicted forced vital capacity, percentage of predicted diffusion capacity of the lung for carbon monoxide, arterial oxygen pressure, Krebs von den Lungen-6, GGO and consolidation on HRCT.

\section{Outcomes and prioritisation}

The primary outcomes of interest will be short-term all-cause mortality and pulmonary-cause mortality, defined as in-hospital mortality or 30-day mortality. The secondary outcomes of interest will include the proportion of patients discharged from the hospital and longterm all-cause mortality, determined at 90 days, 6 months or 1 year after the diagnosis of the disease or the start of treatment. Long-term health-related quality of life will also be considered and will be evaluated according to a validated tool such as the 36-Item Short Form Health Survey. ${ }^{20}$

\section{Studies}

All primary study types (excluding case reports) will be considered for review, provided quantitative data have been used and they describe an association between potential prognostic factors and predefined outcomes for AE of IPF. Furthermore, editorials, letters and review articles will not be considered. Conference proceedings and reports containing abstracts only will not be considered to alleviate concerns of insufficient information. Research papers prior to 2002 will not be considered, as 2002 marked the first year when the current classification 
system of IIPs was first introduced. ${ }^{1}$ Only articles published in English will be reviewed.

\section{Information sources}

- Medline (via Ovid 2002-present).

- EMBASE (via Ovid 2002-present).

- Science Citation Index Expanded (via Web of Science 2002-present).

- Google Scholar.

\section{Search strategy}

Two reviewers (HK and OMP) will search electronic databases, such as Medline and EMBASE using subject headings and text words related to study population such as 'idiopathic pulmonary fibrosis' and 'acute exacerbation'. The Cochrane Database of Systematic Reviews will guide the search process by finding reviews similar to this area of research. Search terms will be combined with methodology filters for prognosis, which can be modified to fit each electronic database (online supplementary appendix).$^{21}{ }^{22}$ The Science Citation Index Expanded will also be consulted using terms adapted from the previous search of Medline and EMBASE. The search period spans 2002 through to 1 April 2019. The reference list of each study eligible for inclusion in this review will also be handsearched to consolidate the implemented search strategy. Grey literature for this subject area will be identified using Google Scholar. ${ }^{23}$

\section{Study records}

\section{Data management}

All retrieved articles will be processed through EndNote $\mathrm{X} 7$, where duplicates can be identified and removed. All extracted data will be stored in a Microsoft Excel spreadsheet.

\section{Study selection and the data collection process}

Two reviewers (HK and OMP) will independently examine the titles and abstracts of all retrieved articles (after removing duplicates) to identify eligible reports. In cases where one research group conducted multiple studies with the same outcome of interest focusing on the same prognostic factor(s), only the study with the largest sample size will be considered. Data will be extracted based on a modified data extraction form used in a previously published protocol paper reviewing prognostic factors. ${ }^{24}$ Any uncertainty or disagreement between reviewers arising from these processes will be resolved by discussion.

\section{Data items}

The following data will be extracted from each eligible study: first author's name, year of publication, study location, study design, sample size (and their demographic features), outcomes of interest, potential prognostic factors for disease, potential aetiology of disease, length of follow-up, methods for statistical analysis, summary statistics and items associated with risk of bias.

\section{Candidate prognostic factors}

Any clinical information relevant to the prespecified outcomes reported by a minimum of three separate studies will be further investigated as potential prognostic factors for this review.

\section{Risk of bias in individual studies}

The Quality in Prognostic Studies (QUIPS) tool will be applied to assess risk of bias in individual studies. QUIPS consists of six domains. Each domain receives an individual bias rating (low, moderate or high), with overall risk of bias based on the combined rating of each domain. For example, a study showing low risk of bias across all domains would be deemed as having low risk of bias overall. ${ }^{25}$

\section{Statistical analysis}

\section{Summary statistics}

Where binary outcomes are presented, effect sizes will be measured using either hazard ratios (HRs) derived from Cox proportional hazards models ${ }^{26}$ or odds ratios (ORs) derived from logistic regression models. ${ }^{27}$ Where an outcome is presented only using a Kaplan-Meier survival curve or log-rank test, HRs will be recalculated, as previously reported. ${ }^{28}$ Where both HRs and log-rank tests are presented, HRs will be prioritised. ORs or risk ratios (RRs) may be calculated manually based on absolute numbers of the outcome of interest across two groups under comparison. Where prognostic factors or the outcome of interest are measured as continuous variables, effect sizes may be presented as absolute values using mean difference (calculated by the unpaired Student's t-test) or difference in medians (calculated by the Wilcoxon rank-sum test).

\section{Data synthesis}

Where an association between one potential prognostic factor and an outcome of interest is presented using the same summary statistics in three or more studies, results will be pooled. Binary outcome will be summarised separately using ORs, RRs or HRs. Continuous outcomes will be combined using mean difference or standardised mean difference (calculated as Hedge's g), ${ }^{29}$ based on whether outcomes are presented using the same unit(s). When the median, range or IQR are presented for continuous variables, they will be converted to a respective mean value with an SD, using a formula reported by a previous study. ${ }^{30}$ Only unadjusted effect estimates for potential prognostic factors will be combined. Effect estimates from multivariate models will be described qualitatively, as model adjustments will likely vary significantly, such that pooling these data could be misleading. If meta-analysis is feasible from the collated data, it will be conducted using a random-effects model employing the DerSimonian and Laird method. ${ }^{31}$ If possible, meta-analysis will be conducted using the Statistical Software Package, Review Manager (RevMan) V.5.3 (Copenhagen: The Nordic Cochrane Centre, The Cochrane Collaboration, 2014). The $95 \%$ prediction interval will be calculated 
if combined results are presented and heterogeneity between studies has been determined.$^{32}$ Statistical significance is considered with respect to a p-value of $<0.05$. If combining data is deemed inappropriate (due to a small number of studies or substantial clinical or methodological variability between studies), results will be reported qualitatively.

\section{Heterogeneity between studies}

Between-study variance will be estimated with respect to the Tau square value and assessed using both $Q$ statistics and the $\mathrm{I}^{2}$ value. For the assessment of heterogeneity between studies, statistical significance will be considered with respect to a $p$-value of $<0.1$ due to the low power of the test. Magnitude of heterogeneity can be categorised as low $(0 \%-30 \%)$, moderate $(30 \%-50 \%)$, considerable $(50 \%-70 \%)$ and substantial $(70 \%-100 \%) .^{29}$ To better interpret sources of heterogeneity, subgroup analysis will be conducted based on the definition of AE of IPF (idiopathic or triggered), study location (Asia or non-Asia) and sample sizes $(n<50$ or $n \geq 50)$. Sensitivity analysis will also be conducted focusing on studies with low risk of bias.

\section{Reporting bias}

Small study bias (such as publication bias) will be examined using graphical asymmetry of a funnel plot and Egger's test, if 10 or more studies are available that report the effect of a specific potential prognostic factor for $\mathrm{AE}$ of IPF. ${ }^{33}$ Statistical significance will be considered with respect to a $p$-value of $<0.1$ due to the low power of the test. If publication bias is suspected, an adjusted summary effect will be estimated using the trim and fill method, which considers the presumptive number of missing studies. ${ }^{34}$

\section{Confirmation of prognostic factors}

Prognostic factors will be determined and judged based on statistically significant findings and the consistency of results. Prognostic factors will be confirmed if their effects are consistently in the same direction across all studies and statistically significant in at least $75 \%$ of the included studies. Effects from multivariate analyses will be considered for confirmation of prognostic factors.

\section{Confidence in cumulative evidence}

The credibility of evidence generated from this systematic review will be assessed by the Grades of Recommendation, Assessment, Development and Evaluation (GRADE) system. The GRADE system will be applied to the final list of confirmed prognostic factors generated from both univariate and multivariate results. ${ }^{35}$

\section{ETHICS AND DISSEMINATION}

Extensive ethical consideration will not be required to conduct this systematic review as evidence will be generated from existing published data. Furthermore, patientlevel or potentially identifiable information will not be accessed. The results of the review will be reported according to Preferred Reporting Items for Systematic Reviews and Meta-Analyses ${ }^{36}$ and Meta-analysis of Observational Studies in Epidemiology guidelines. ${ }^{37}$ A Microsoft Excel spreadsheet containing all data gathered for this review will be stored in a digital repository such as Dryad for open access after publication and may be made available on reasonable request to the corresponding author.

\section{DISCUSSION}

This article has outlined the rationale for a methodologically sound systematic review of prognostic factors for $\mathrm{AE}$ of IPF. Due to the relative complexity of conducting systematic reviews of prognostic factors, ${ }^{38}$ a detailed description of the proposed methodology was required to ensure transparency and research integrity for the proposed study. ${ }^{39}$

There are several methodological limitations that warrant discussion to appropriately interpret the findings of this proposed study. First, prognostic factors will be determined based on the result of multivariate analysis, which will be summarised qualitatively in this review. This may result in the omission or misclassification of potential prognostic factors due to the low power of individual studies with small sample sizes. Statistical synthesis is expected to solve this issue. ${ }^{40}$ However, pooling multivariate data can be misleading as adjusted variables and the final model will be diverse between studies. ${ }^{41}$ Besides, prognostic factors will be determined based on statistically significant results and the consistency of findings. This is an arbitrary measure that may disregard other potentially viable prognostic factors for the disease. Therefore, even if some potential prognostic factors are not confirmed in this proposed study, we suggest that all identified factors be examined for their clinical significance in future research. Furthermore, the results of this proposed study should be updated to include future research.

Second, it is likely that studies identified for this review will be both clinically and methodologically heterogeneous. The included studies may contain a mix of patients with both idiopathic and triggered forms of $\mathrm{AE}$ of IPF, diagnosed using the previous (narrow) ${ }^{6}$ or current (broad) diagnostic criteria. ${ }^{7}$ Additionally, the definition of an outcome may also vary between studies. For example, mortality may be evaluated at different timescales across studies, such as in-hospital, 30 days, 90 days or 1 year after the diagnosis of the disease or the start of treatment. Comparison of outcomes may be further complicated for continuous factors, which could be categorised with arbitrary cut-off points, imposed by each respective research group. ${ }^{42}$ While these limitations may undermine some of the statistical capabilities of the proposed meta-analysis, a qualitative description of results may also provide meaningful insights into prognostic factors for AE of IPF.

Finally, potential prognostic factors will be selected for further analyses if they are reported in a minimum 
of three separate studies. Repeated mention of clinical information may suggest clinical relevance, which could serve to improve the applicability of our findings. By employing this inclusion criteria, potential prognostic factors reported by only one or two studies will be omitted. This will deprive these potential prognostic factors from further investigation in this study and may stifle their further research in other studies.

Despite the potential methodological limitations discussed in this protocol paper, we believe in the value of clarifying current evidence surrounding prognostic factors for AE of IPF through systematic review. Peer review of this protocol paper will also serve to improve the integrity and transparency of our proposed research.

\section{CONCLUSION}

This protocol paper outlined the need for a methodologically sound systematic review of prognostic factors for $\mathrm{AE}$ of IPF. The methodological limitations of the proposed study are common to research examining prognostic factors and are largely unavoidable. Despite these limitations, this study would represent the leading body of evidence for this area of research.

Acknowledgements We would like to thank Mr Istvan T Kabdebo of The School of Population and Global Health, University of Western Australia, for his support in editing this manuscript.

Contributors HK conceptualised this research project and its associated methodology. HK also wrote the manuscript for this protocol. HK will be the guarantor of the content of the review including data analysis. OMP contributed in the conceptualisation of this research project by planning the literature search strategy and data extraction methods. OMP also made additions and revisions to the draft of this manuscript.

Funding The authors have not declared a specific grant for this research from any funding agency in the public, commercial or not-for-profit sectors.

Competing interests None declared.

Patient consent for publication Not required.

Provenance and peer review Not commissioned; externally peer reviewed.

Open access This is an open access article distributed in accordance with the Creative Commons Attribution Non Commercial (CC BY-NC 4.0) license, which permits others to distribute, remix, adapt, build upon this work non-commercially, and license their derivative works on different terms, provided the original work is properly cited, appropriate credit is given, any changes made indicated, and the use is non-commercial. See: http://creativecommons.org/licenses/by-nc/4.0/.

\section{REFERENCES}

1. American Thoracic Society/European Respiratory Society International Multidisciplinary Consensus Classification of the Idiopathic Interstitial Pneumonias. This joint statement of the American Thoracic Society (ATS), and the European Respiratory Society (ERS) was adopted by the ATS board of directors, June 2001 and by the ERS Executive Committee, June 2001. Am J Respir Crit Care Med 2002:277-304.

2. Raghu G, Chen SY, Yeh WS, et al. Idiopathic pulmonary fibrosis in US Medicare beneficiaries aged 65 years and older: incidence, prevalence, and survival, 2001-11. Lancet Respir Med 2014;2:566-72.

3. Kärkkäinen M, Nurmi H, Kettunen HP, et al. Underlying and immediate causes of death in patients with idiopathic pulmonary fibrosis. BMC Pulm Med 2018;18:69.
4. Kim DS, Park JH, Park BK, et al. Acute exacerbation of idiopathic pulmonary fibrosis: frequency and clinical features. Eur Respir J 2006;27:143-50.

5. Ley B, Collard HR, King TE. Clinical course and prediction of survival in idiopathic pulmonary fibrosis. Am J Respir Crit Care Med 2011;183:431-40.

6. Collard HR, Moore BB, Flaherty KR, et al. Acute exacerbations of idiopathic pulmonary fibrosis. Am J Respir Crit Care Med 2007;176:636-43.

7. Collard HR, Ryerson CJ, Corte TJ, et al. Acute Exacerbation of Idiopathic Pulmonary Fibrosis. An International Working Group Report. Am J Respir Crit Care Med 2016;194:265-75.

8. Agarwal R, Jindal SK. Acute exacerbation of idiopathic pulmonary fibrosis: a systematic review. Eur J Intern Med 2008;19:227-35.

9. Huie TJ, Olson AL, Cosgrove GP, et al. A detailed evaluation of acute respiratory decline in patients with fibrotic lung disease: aetiology and outcomes. Respirology 2010;15:909-17.

10. Juarez MM, Chan AL, Norris AG, et al. Acute exacerbation of idiopathic pulmonary fibrosis-a review of current and novel pharmacotherapies. J Thorac Dis 2015;7:499-519.

11. Song JW, Hong SB, Lim CM, et al. Acute exacerbation of idiopathic pulmonary fibrosis: incidence, risk factors and outcome. Eur Respir $J$ 2011;37:356-63.

12. Richeldi L. Time for Prevention of Idiopathic Pulmonary Fibrosis Exacerbation. Ann Am Thorac Soc 2015;12(Suppl 2):S181-5.

13. Atsumi K, Saito Y, Kuse N, et al. Prognostic Factors in the Acute Exacerbation of Idiopathic Pulmonary Fibrosis: A Retrospective Single-center Study. Intern Med 2018;57:655-61.

14. Simon-Blancal V, Freynet $\mathrm{O}$, Nunes $\mathrm{H}$, et al. Acute exacerbation of idiopathic pulmonary fibrosis: outcome and prognostic factors. Respiration 2012;83:28-35.

15. Kamiya H, Panlaqui OM, Izumi S, et al. Prognostic factors of idiopathic inflammatory myopathies complicated with interstitial lung disease: protocol for a systematic review and meta-analysis. BMJ Open 2016;6:e012744.

16. Centre for Reviews and Dissemination, University of York. PROSPERO: International Prospective Register of Systematic Reviews http://www.crd.york.ac.uk/prospero/ (Accessed 19 Nov 2018).

17. Raghu G, Collard HR, Egan JJ, et al. An official ATS/ERS/JRS/ALAT statement: idiopathic pulmonary fibrosis: evidence-based guidelines for diagnosis and management. Am J Respir Crit Care Med 2011;183:788-824.

18. Gono T, Kawaguchi $Y$, Satoh $T$, et al. Clinical manifestation and prognostic factor in anti-melanoma differentiation-associated gene 5 antibody-associated interstitial lung disease as a complication of dermatomyositis. Rheumatology 2010;49:1713-9.

19. Inokuma S. Leflunomide-induced interstitial pneumonitis might be a representative of disease-modifying antirheumatic drug-induced lung injury. Expert Opin Drug Saf 2011;10:603-11.

20. Ware JE, Snow KK, Kosinski M, et al. SF-36 Health Survey Manual and Interpretation Guide. Boston, MA: The Health Institute, New England Medical Center, 1993.

21. Wilczynski NL, Haynes RB; Hedges Team. Developing optimal search strategies for detecting clinically sound prognostic studies in MEDLINE: an analytic survey. BMC Med 2004;2:23.

22. Wilczynski NL, Haynes RB. Optimal search strategies for detecting clinically sound prognostic studies in EMBASE: an analytic survey. $J$ Am Med Inform Assoc 2005;12:481-5.

23. Haddaway NR, Collins AM, Coughlin D, et al. The Role of Google Scholar in Evidence Reviews and Its Applicability to Grey Literature Searching. PLoS One 2015;10:e0138237.

24. Kamiya H, Panlaqui OM. Prognostic significance of autoantibodies for idiopathic pulmonary fibrosis: protocol for a systematic review. BMJ Open 2018;8:e020862.

25. Hayden JA, Côté P, Bombardier C. Evaluation of the quality of prognosis studies in systematic reviews. Ann Intern Med 2006;144:427-37.

26. Symons MJ, Moore DT. Hazard rate ratio and prospective epidemiological studies. J Clin Epidemiol 2002;55:893-9.

27. Bock JR, Afifi AA. Estimation of probabilities using the logistic mode in retrospective studies. Comput Biomed Res 1988;21:449-70.

28. Tierney JF, Stewart LA, Ghersi D, et al. Practical methods for incorporating summary time-to-event data into meta-analysis. Trials 2007;8:16.

29. Higgins JPT, Green S. The Cochrane Collaboration. Cochrane Handbook for Systematic Reviews of Interventions Version 5.1.0 [updated March 2011]. 2011 http://www.handbook.cochrane.org

30. Wan X, Wang W, Liu J, et al. Estimating the sample mean and standard deviation from the sample size, median, range and/or interquartile range. BMC Med Res Methodol 2014;14:135. 
31. DerSimonian R, Laird N. Meta-analysis in clinical trials. Control Clin Trials 1986;7:177-88.

32. Riley RD, Higgins JP, Deeks JJ. Interpretation of random effects meta-analyses. BMJ 2011;342:d549.

33. Egger M, Davey Smith G, Schneider M, et al. Bias in meta-analysis detected by a simple, graphical test. BMJ 1997;315:629-34.

34. Duval S, Tweedie R. Trim and fill: A simple funnel-plot-based method of testing and adjusting for publication bias in meta-analysis. Biometrics 2000;56:455-63.

35. Iorio A, Spencer FA, Falavigna M, et al. Use of GRADE for assessment of evidence about prognosis: rating confidence in estimates of event rates in broad categories of patients. BMJ 2015;350:h870.

36. Moher D, Liberati A, Tetzlaff J, et al. Preferred reporting items for systematic reviews and meta-analyses: the PRISMA statement. Ann Intern Med 2009;151:264-9.

37. Stroup DF, Berlin JA, Morton SC, et al. Meta-analysis of observational studies in epidemiology: a proposal for reporting.
Meta-analysis Of Observational Studies in Epidemiology (MOOSE) group. JAMA 2000;283:2008-12.

38. Altman DG. Systematic reviews of evaluations of prognostic variables. BMJ 2001;323:224-8.

39. Tsujimoto Y, Tsujimoto H, Kataoka Y, et al. Majority of systematic reviews published in high-impact journals neglected to register the protocols: a meta-epidemiological study. J Clin Epidemiol 2017;84:54-60.

40. Crowther M, Lim W, Crowther MA. Systematic review and metaanalysis methodology. Blood 2010;116:3140-6.

41. Fisher AV, Fernandes-Taylor S, Campbell-Flohr SA, et al. 30-day Readmission After Pancreatic Resection: A Systematic Review of the Literature and Meta-analysis. Ann Surg 2017;266:242-50.

42. Royston P, Altman DG, Sauerbrei W. Dichotomizing continuous predictors in multiple regression: a bad idea. Stat Med 2006;25:127-41. 